\title{
Thin film HF RFID tag deposited on paper by thermal evaporation
}

\section{Camille Ramade*, Sébastien Silvestre, Frédérique Pascal-Delannoy and Brice Sorli}

Institut d'Electronique du Sud (IES),

UMR CNRS 5214,

Université Montpellier 2,

Place E. Bataillon, Bat 21 CC075

34095 Montpellier Cedex 05, France

Email: camille.ramade@ies.univ-montp2.fr

Email: sebastien.silvestre@ies.univ-montp2.fr

Email: frederique.pascal-delannoy@ies.univ-montp2.fr

Email: brice.sorli@ies.univ-montp2.fr

*Corresponding author

\begin{abstract}
The authors describe for the first time the fabrication and characterisation of an HF Radio Frequency Identification (RFID) transponder (tag) deposited on paper substrate using thermal evaporation. This technique is well adapted to the depositing of thin layers with good electrical properties directly on paper. This process helps to reduce the required amount of metal and the number of manufacturing stages in the realisation of RFID labels. The coil antenna consists of a thin aluminium layer evaporated through a masking system. The low thickness of metal that grows during thermal evaporation presents a good metal conductivity. After the RFID chip bonding, the tags are perfectly operational. We have developed the theoretical aspect related to the parameter thickness of the layer deposited as well as the simulation of the transponder. Technological processes implemented to carry out the transponder are then described. The electrical characterisations of the tags are presented and their good performance is illustrated.
\end{abstract}

Keywords: RFID; radio frequency identification; high frequency; tags; antenna; paper; thermal-evaporation; aluminium.

Reference to this paper should be made as follows: Ramade, C., Silvestre, S., Pascal-Delannoy, F. and Sorli, B. (2012) 'Thin film HF RFID tag deposited on paper by thermal evaporation', Int. J. Radio Frequency Identification Technology and Applications, Vol. 4, No. 1, pp.49-66.

Biographical notes: Camille Ramade currently works at the Laboratory of the Institut d'Electronique du Sud, Montpellier University on the New Technologies related to RFID. He received his MS degree in Electrical Engineering and Microwave from Montpellier University in 2008. Since then he has been preparing a professional Doctorate of Electronic at the Graduate School I2S in Montpellier. 
Sébastien Silvestre is currently working on a new method for achieving low cost RFID tags. He his MS degree in Electrical Engineering and Microwave from Montpellier University in 2007. Since then he has been working in the Laboratory of the Institut d'Electronique du Sud, Montpellier University.

Frédérique Pascal-Delannoy received her $\mathrm{PhD}$ in Electrical Engineering from Montpellier University in 1988. Since then she has been working at the Laboratory of Electronic and Micro-Optoelectronic Center of Montpellier renamed in 2006 as 'Institut d'Electronique du Sud', Montpellier University. She is an Specialist of thin film materials grown using different techniques (MOCVD, sputtering deposition, thermal evaporation). Until now, she has been involved in preparation and study of low size sensors based on thermal transport phenomena. Since 2006, she has been working on sensor applications in RFID.

Brice Sorli received his MS degree, in Electrical Engineering, and $\mathrm{PhD}$ degree both from Montpellier University in 1998 and 2001, respectively. During this period, he worked on electronic measurements, instrumentation, thermal analysis and humidity sensors. In 2002, he joined the Laboratoire d'Electronique et de Nanotechnologies Capteurs, Claude Bernard University, Lyon, where he was involved in the designing and implementation of nuclear magnetic resonance micro-probe for 'Labs on chip' and in vivo applications. In 2005, he joined l'Institut d'Electronique du Sud, Montpellier University, and currently works on the sensors applications in RFID.

\section{Introduction}

The use of Radio Frequency Identification (RFID) systems has considerably increased recently. RFID technologies are used, for example, in logistics and access control. The communication protocol and coupling are based on electromagnetic waves and fields. Before RFID, barcodes on product packages and magnetic strips on bank and security cards were the main means of identification and tracking in transactions. RFID alternative technologies provide an automatic means of delivering product data without direct contact between the RFID label, tag or transponder and the reader device. This technology aims to gradually replace a significant portion of other tracking and access devices. Most of RFID applications are in the Low Frequency (LF), High Frequency (HF) and Ultra High Frequency (UHF) ranges such as $125 \mathrm{kHz}, 13.56 \mathrm{MHz}$ and $868 \mathrm{MHz}$, respectively (Finkenzeller, 2003; Paret, 2003).

HF labels are widely used in RFID systems. As shown in Figure 1, they consist of four main components (Hunt, 2007):

- a substrate that can be Polyethylene Terephthalate (PET), PVC or paper as in our case

- a metallic coil that we call 'antenna' made of copper, aluminium or sometimes conductive ink

- a RFID chip or a strap mounted on the antenna, which stores data

- an overlay PVC, epoxy, adhesive, depending on application requirements. 
Figure 1 RFID transponder structure from Hunt (2007)

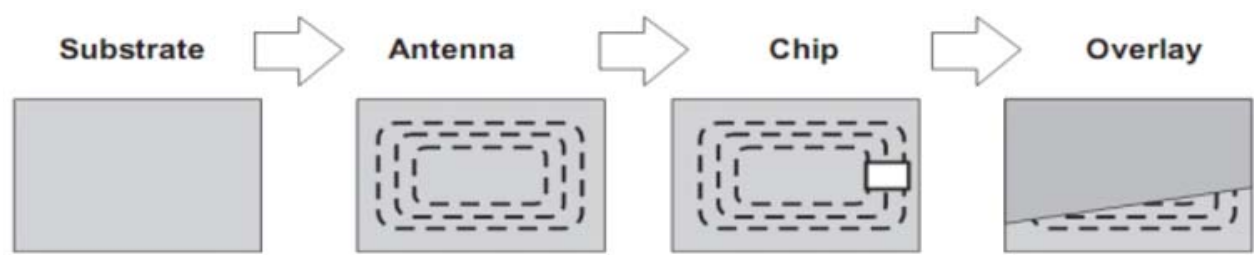

Various types of RFID transponders exist, some have a battery, such as active or batteryassisted tags, and other are without battery. These battery-less transponders are called passive tag and all electrical power required for the communication and chip must be brought through the electromagnetic radiation emitted by the reader (Hunt, 2007).

At present, the price of an RFID tag is still high compared to the barcode and, due to the large volume of individual packages, the cost of the antenna of this tag must be taken into consideration. In this context, paper substrate is of tremendous economic importance, as it is cheap, environmentally friendly and widely used in packages. In the semiconductor industry, very little research has been carried out on this organic substrate (Eder et al., 2004; Fortunato and Correia, 2008). In the RFID field, transponders are generally built on flexible polyester film such as PET. Paper is a potential substrate on which the tag can be directly printed (Ferrer-Vidal et al., 2006; Merilampi et al., 2007; Yang et al., 2007) and fabricated in high volumes. This makes paper the most costeffective material for this application.

There are several techniques used to deposit an antenna on PET: etching, electroplating; and on paper: screen printing, flexography and offset (lithography) (Paret, 2003). In order to develop a new process of RFID tag manufacturing, the thermal evaporation technique has been improved for growing metallic film directly on PET or paper substrates. This technique is well-known in microelectronics (Umrath, 1998) and is already used industrially for packaging metallisation and for capacitor web coating. This coating is carried out with industrial machines called 'metalisers' that run with a highspeed roll-to-roll process under vacuum (Ludwig, 2005). It allows to obtain a thin and homogeneous metal layers with a high electrical conductivity. Thermal evaporation offers numerous advantages: firstly this is a 'dry' and fast technique, without baths or drying stages. This is possible thanks to a quick and reusable masking system. Secondly, the use of aluminium as material is economical compared to copper or silver inks which are both very expensive. Finally, it is possible to work with a paper substrate that is ecological, economical, printable and easily convertible into labels without any additional process stages.

As this technique requires only a small quantity of metal, the use of paper substrate and a fast masking stage, the mass production costs of tags prove to be low, thus confirming the viability of this study.

As a summary, the Joule evaporation technique offers:

- $\quad$ low metal consumption (aluminium)

- $\quad$ low-cost substrate (paper)

- fast, accurate and reproducible designs

- $\quad$ less manufacturing stages. 
To start we have presented in Section 2 the existing techniques to manufacture RFID label to compare with our proper technological process implemented to build the transponder. Then the paper approaches the theoretical considerations taking into account the technology of HF RFID coil with low film thickness in Section 3. The electromagnetic simulation of the tag and measurements are then explained in Section 4. Lastly, the prototype is tested using a Tagtester, a network analyser and a commercial reader before discussing the results.

\section{Experimental details}

\subsection{Techniques of RFID tags fabrication}

This section describes the most used manufacturing techniques for producing RFID tags. For each method, the process, advantages and disadvantages are briefly presented. The technique of thermal evaporation by Joule effect is also described and positioned to other methods. Then, the industrialisation process investigated for the metallisation by evaporation in large volume is discussed.

\subsubsection{Etching}

The etching is currently the oldest and most used technique for the production of RFID tag antennas. This is a subtractive process where a part of metal which constitutes the antenna is etched. It is widely implemented in the realisation of printed electronic circuits. This process is carried out in several steps from a substrate on which is rolled or electrodeposited uniformly a conductor layer, usually copper or aluminium from 10 to $20 \mu \mathrm{m}$ thick. Then a mask is made on this layer to protect the areas that will be kept. The mask is made by photolithography using a photosensitive resin or a screen printing. Finally, the unprotected conductive parts are removed in a chemical solution bath. There are two variants of the etching process: the dry and the wet which have been described in more details by Elsherbeni et al. (2010). The wet process permits to attain good resolution and antenna performance but it needs lot of steps and is incompatible with paper substrate and obliged to use expensive polymer substrates like PET, PVC or PC.

\subsubsection{Electroplating}

The plating is a technique commonly used in industry for making metal layers of varying thickness. This consists of metal particles to migrate toward a target, usually a metal surface with the use of a chemical electrolytic bath. In the case of RFID the starting substrate is PET with a bonding layer of several tens of nanometres of aluminium, chromium or nickel on which copper layer will grow. The growth of copper plating is done in an electrolytic bath after masking the target zone. This is an additive process which permits to control the amount of metal used but which is also very slow, quite expensive and incompatible with paper substrate. 


\subsubsection{Printing - flexo, helio, screen printing, offset}

Printing is an additive process that is widely used in the manufacture of RFID tags. There are several printing techniques for producing RFID antennas using conductive inks: screen printing, flexography, rotogravure, inkjet and offset. This technique permits to use paper as substrate; however, it has many disadvantages: a slow step of ink drying, a low resolution of the pattern for some of them, an expensive price of the ink charged with silver particles and a low value of conductivity which affects the antenna performance if the thickness of conductive ink is not sufficient. Currently new conductive inks are being studied to improve the performance of printing techniques. These inks are made of metal particles 100 to $0.1 \mu \mathrm{m}$ in suspension in various polymer resins. Many studies and publications describe the use of printing technologies to produce RFID antennas (Ferrer-Vidal et al., 2006; Merilampi et al., 2007; Yang et al., 2007).

\subsubsection{Stamping}

The stamping is a process for producing RFID tag recently developed by some manufacturers. The principle is quite simple: an aluminium sheet of several tens of microns is glued onto a PET substrate, then a die-cutting tool engraved with the antenna design is used to cut the aluminium layer without cutting the substrate. The next step is to pull and remove the unnecessary part of the aluminium layer while keeping the antennas made onto the substrate. This process is very fast but uses expensive material with a large amount of aluminium waste and does not permit to attain sufficient precision for the major part of RFID antennas. Thus, the stamping method is only used for the production of very simple UHF dipole antennas.

\subsubsection{Evaporation}

The thermal evaporation is a method commonly used in laboratories to produce layers of a few nanometres to a few micrometres thick (Umrath, 1998; Sze, 2001). This method is already widely used industrially for producing packaging with insulation made of aluminium. In the case of RFID antennas production, it is based on copper or aluminium evaporated under vacuum on a substrate using various masking systems more or less complex. The evaporated metal atoms are deposited onto the unmasked areas, thereby constituting an homogeneous metallic layer representing the antenna. This technique and the equipment used in our laboratory are described in details later in this paper.

The main disadvantage of this method is the small thickness of metal deposited which subsequently complicates the matching between the antenna and the chip. However, the purity of the deposited material offers a good electrical conductivity that compensates this inconvenient. Furthermore, the process is simple and does not require lot of manufacturing steps. In fact, to transform the RFID inlay onto a printable label all the techniques previously described except printing and evaporation process need to use additional layers of adhesive and paper which causes a supplementary manufacturing step. The evaporation permits to produce the antenna directly onto a printable paper substrate without any additional step. The resolution of the antennas is sufficient depending on the masking system used. It is also more environment friendly because it is based on paper substrate and thin aluminium layer and does not use harmful chemicals 
products such as solvents or acids during the production process. This last ecological aspect is currently largely described in the literature and shows the interest to develop new techniques of RFID production more environment friendly (Wager et al., 2005).

\subsubsection{Industrialisation of thermal evaporation (vacuum coating)}

For large-scale manufacturing of RFID tags, the method of metallisation by thermal evaporation must be easily transferable to industrial machines. These equipments already exist as shown in the articles of Ludwig (2005) and Olbrich (2005) especially for the production of films capacities deposited on flexible substrates such as PET and paper. Figure 2 shows a view of similar equipment from Multimeter company dedicated to this application. These metalising systems are generally composed of a pumping system which produces a high vacuum in the chamber where we could find the winding system, the masking system, the process control and the evaporator modules. All these parts are controlled simultaneously by a control cabinet. For RFID antenna production, some improvements will be necessary to adapt the masking system to our need. This production is carried out with industrial machines called 'metalisers' which run with a high-speed roll-to-roll process under vacuum $(10 \mathrm{~m} / \mathrm{min})$ and enables production of several thousand of antenna in few hours with good reproducibility. We already tested similar industrials equipments and produced some reel with one of this metaliser to confirm the feasibility of industrial RFID production by thermal evaporation. Figure 3 shows an example of this result, it represents a small part of a $500 \mathrm{~m}$ roll antenna produced with a metaliser and consisted of different HF designs. These antennas are made directly on paper with the metaliser roll-to-roll process and composed of a $350 \mathrm{~nm}$ thick layer of aluminium.

Figure 2 Schematic of film metalising system (metaliser) (see online version for colours)

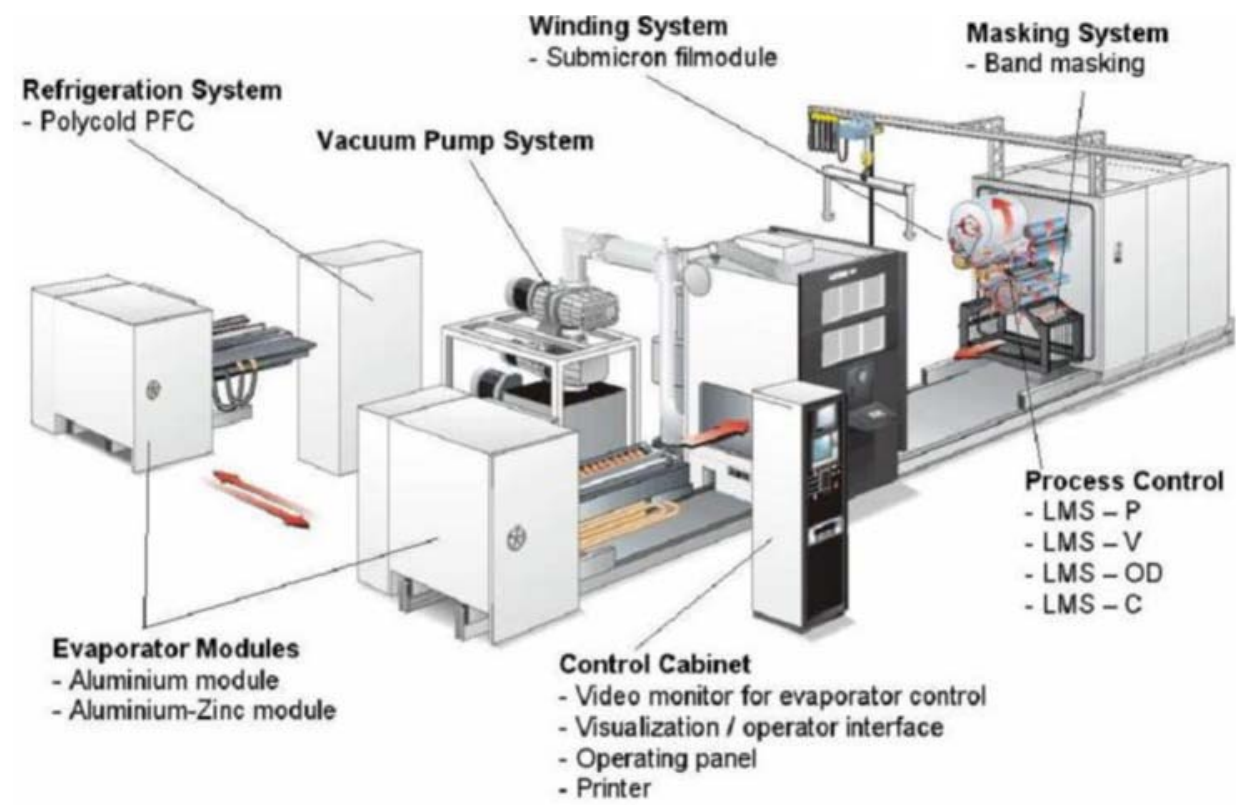


Figure 3 HF RFID antenna roll produced industrially with a metaliser (see online version for colours)

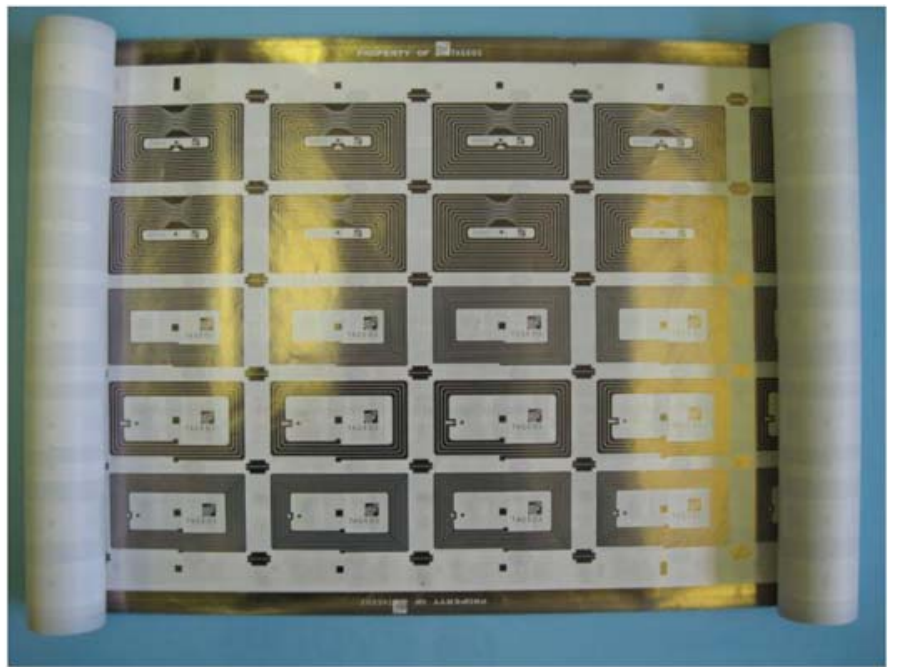

\subsection{Realisation}

The HF tags are fabricated in two stages: the antenna is initially deposited on paper by thermal evaporation then the RFID chip is fixed by thermocompression.

\subsubsection{Evaporation process}

Thermal evaporation is one of the most commonly used metal depositing techniques (Umrath, 1998). It consists of vaporising a solid material by heating it to a sufficiently high temperature and recondensing it onto a substrate to form a thin metallic film. This technique is well adapted to form the coil antenna directly on paper substrate. As shown in Figure 4, our evaporation equipment comprises a pumping system used to create a high vacuum in the glass bell jar, a tungsten boat connected to a high current source, a substrate table placed vertically up against the thermal resistive source boat and a quartz balance for the measurement of growth rate and metal thickness deposited.

Our laboratory masking system is composed of a $200 \mu \mathrm{m}$ thick nickel mask created with electro-deposition by the company Stork. This mask is removable and reusable thanks to its thickness and rigidity. It is placed on a positioning support with guide lines to maintain the gap between tracks; the paper is then placed on the aligned mask. To finish we use a magnetic support to maintain the mask in position against the paper before removing the positioning support. After the masking stage, we evaporate extremely pure aluminium wire $(99.9 \%)$ through the mask directly onto the paper surface.

During the evaporation process, the vacuum pressure is maintained at $5.10^{-5}$ Torr, the thickness of deposited metal $(300-1000 \mathrm{~nm})$ is controlled by the quartz balance. The deposition rate ranges between 2 and $5 \mathrm{~nm} / \mathrm{s}$ corresponding to an evaporation time of 1-4 minutes. During this time, the paper is exposed to a thermal radiation varying between 60 and $70^{\circ} \mathrm{C}$. This value is measured with a thermal indicator. 
Figure 4 Schematic diagram of a resistive thermal evaporation system

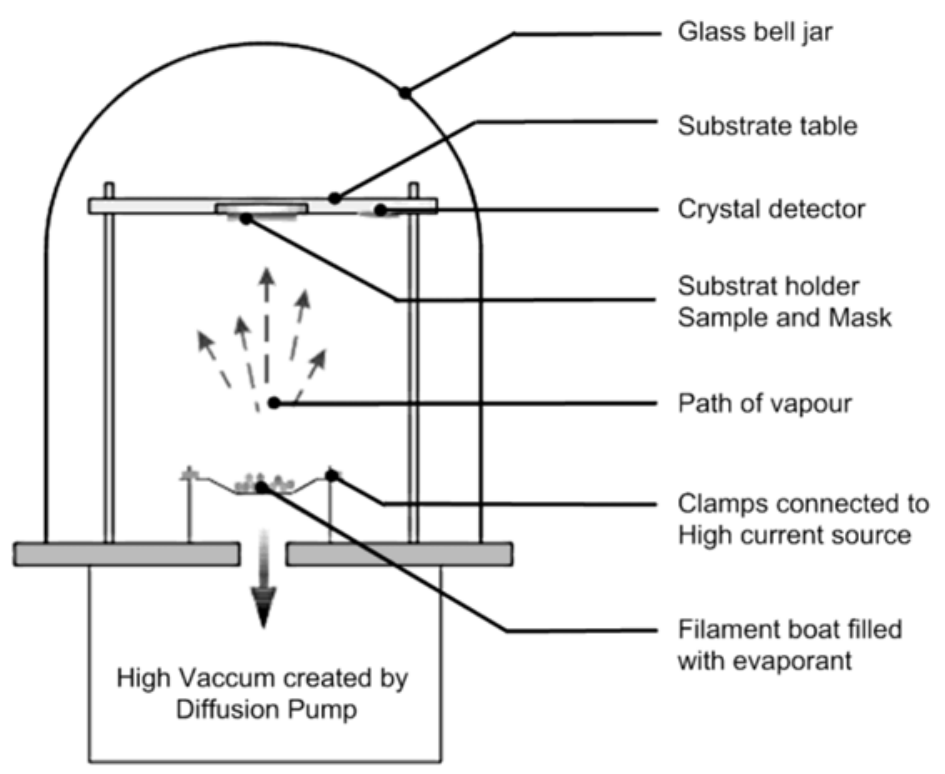

This process is already industrially improved by our industrial partner and the post production has been launched successfully. We currently optimised this process to increase the productivity and the tags performances.

\subsubsection{Paper treatment}

Several types of paper substrate were used: vellum papers as well as single or double coated papers, all of which have the same weight of $80 \mathrm{~g} / \mathrm{m}^{2}$, a thickness of $76 \mu \mathrm{m}$ and dielectric constant close to 1.2 (manufacturer's data). The paper substrate offers many advantages: economical, ecological and printable. However, it is difficult to work as its fibrous makes the paper substrate particularly fragile, it is quite rough and there is a risk of heating or degassing. We therefore use a varnish composed of a styrene-acrylic emulsion deposited by flexography on the surface of the paper substrate $\left(1 \mathrm{~g} / \mathrm{m}^{2}\right.$ dry $)$. This varnish solves the problems related to the use of paper substrate for vacuum thermal metallisation. The surface is smooth and the paper substrate resists high temperature up to $150^{\circ} \mathrm{C}$ for several minutes. Moreover, this varnish helps to reduce out-gassing.

\subsubsection{Chip connection}

The next stage involves connection of the RFID chip to the antenna. The selected proximity chip is the Mifare ${ }^{\circledR}$ MF1 from NXP. These chips are proposed by manufacturers in wafer form or on reels of straps. The strap solution is interesting as it takes the place of the bridge, thus makes the industrialisation process faster and easier. The latter packaging method was selected for the realisation of our HF transponder. The strap is then stuck by thermo-compression using a Mühlbauer Thermode Test Station (TTS 300) with a conductive adhesive. 


\section{Theoretical considerations}

\subsection{General theory}

The classic equivalent circuit of the tag is represented in Figure 5. This is an RLC structure operating at the RFID frequency of $13.56 \mathrm{MHz}$. One finds the part corresponding to the chip with its capacitance $C_{i c}$ and its internal resistance $R_{i c}$. The coil, also referred to as 'antenna', is represented by its resistance $R_{\text {ant }}$ and its inductance $L_{\text {ant }}$. Finally the sum of the parasitic capacitances related to the strap and the connection of the chip to the antenna is represented by $C_{c o n}$.

Figure 5 Electrical equivalent schematic of transponder

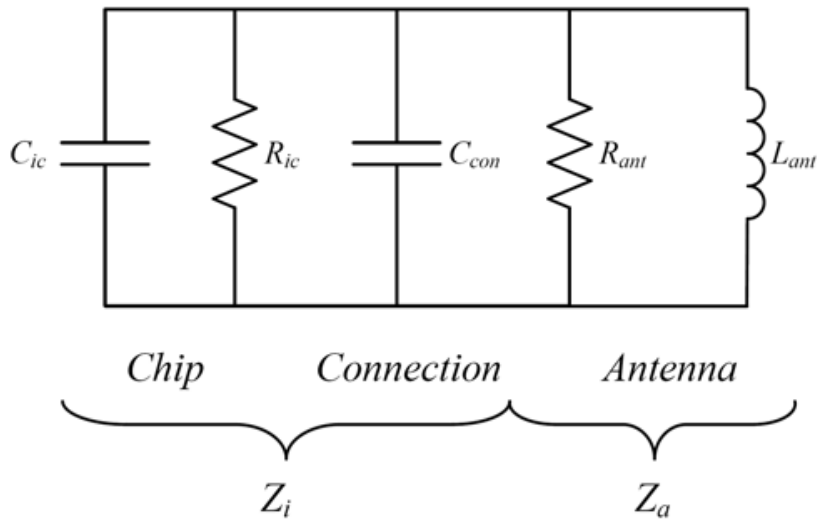

In order to optimise the energy transfer between the antenna and the chip, it is imperative that the antenna impedance $Z_{a}$ matches the chip and its connection impedance $Z_{i}$. 'So the impedances of the antenna and the chip must be matched one to the other [equation (1)], the real parts must be equal [equation (2)] and the imaginary parts opposites [equation (3)]':

$$
\begin{aligned}
& Z_{a}=Z_{i}^{*} \\
& R_{a n t}=R_{i c} \\
& X_{a}=j L_{a n t} \omega=-X_{i} \\
& f=\frac{1}{2 \pi \sqrt{L C}}
\end{aligned}
$$

As the chip impedance is essentially capacitive, the antenna of the tag must be of the selfinductive type. Knowing the various values of capacities given by the manufacturers and measurements, it remains to determine the value of the antenna inductance $(L)$ required for matching at the operating frequency $13.56 \mathrm{MHz}$. In our case, for an internal capacitance $\left(C_{i c}\right)$ equal to $16 \mathrm{pF}$ and a connection $\left(C_{c o n}\right)$ of $2 \mathrm{pF}$, the antenna must have an inductance of approximately $7 \mu \mathrm{H}$ and a serial resistance close to $50 \Omega$ to achieve a sufficient matching and to produce a resonance at the operating frequency. 


\subsection{Calculation of coil inductance}

The coil antenna is composed of a conductive multi-turn spiral which is equivalent to an RF inductance represented in Figure 6. With application notes provided by the chip manufacturer (Philips Semiconductors, 2006) and RFID literature (Paret, 2003), we can determine the theoretical value of the serial inductance needed to match this antenna to the chip and the connection. The parameters necessary to compute are the structural dimensions of the coil and some factors related to the mutual inductance.

$$
L_{\text {sant }}=4(a+b)\left[\ln \left(\frac{2(a+b)}{w}\right)-k\right] N^{p}
$$

For example, using antenna dimensions listed in Table 1 with external dimensions ( $a$ and $b$ ) corresponding to the HF RFID standard, we obtain analytically an inductance equal to $7 \mu \mathrm{H}$ [equation (5)]. This value corresponds to the one previously determined for correct matching of the antenna to the chip [equation (3)]. Therefore, we will use these typical values to fabricate our HF prototype tag.

Figure 6 RFID HF coil antenna structure

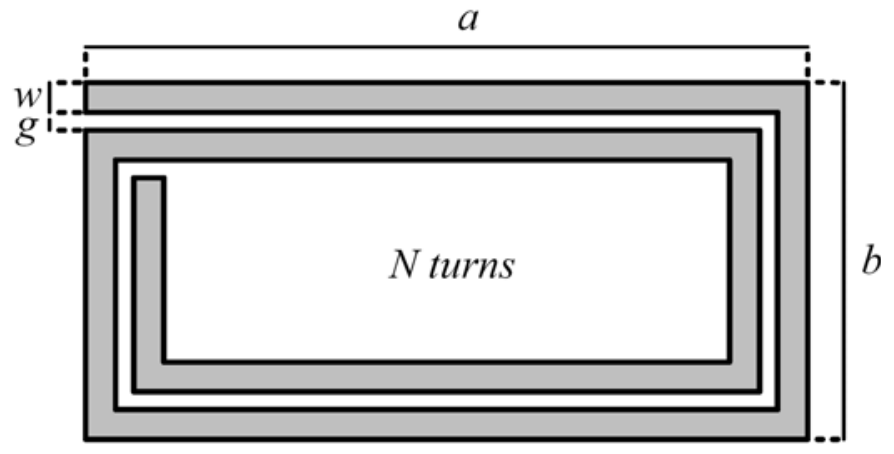

Table 1 Units for inductance calculation

\begin{tabular}{llc}
\hline Symbol & \multicolumn{1}{c}{ Quantity } & Example values \\
\hline $\mathrm{a}$ & Length of structure & $75 \mathrm{~mm}$ \\
$\mathrm{~b}$ & Width of structure & $45 \mathrm{~mm}$ \\
$\mathrm{w}$ & Track width & $1.1 \mathrm{~mm}$ \\
$\mathrm{~g}$ & Gap between tracks & $0.2 \mathrm{~mm}$ \\
$\mathrm{t}$ & Track thickness & $0.3-1 \mu \mathrm{m}$ \\
$\mathrm{N}$ & Number of turns & 10 \\
$\mathrm{k}$ & Geometrical factor & 1.04 (for rectangular coil) \\
$\mathrm{p}$ & Technologic factor & 1.85 (for evaporated coil) \\
\hline
\end{tabular}




\subsection{Calculation of coil resistance}

Referring to the electrical equivalent schematic of the transponder shown in Figure 5 and the conditions of matching, it is necessary to adapt the real part of the antenna to the internal resistance of the RFID chip: $R_{a}=R_{i}$ [equation (2)].

Due to the low thickness of deposited material $(0.3-1 \mu \mathrm{m})$, matching of the real part is quite difficult to achieve. The design of the antenna can be modified to maintain the inductance high enough (imaginary part) while correctly adapting the real part. Knowing the length of the coil $l$, the width of the track $w$, the deposited thickness $t$ and conductivity of the material $\sigma$, it is possible to establish the equivalent serial resistance of the coil:

$$
R_{\text {sant }}=\frac{1}{\sigma t w}
$$

Figure 7 shows the variation of the coil inductance and resistance as a function of the thickness for three different conductive materials commonly used in RFID: copper [Cu], aluminium [Al], silver ink [Ag] with respective conductivities measured of: $\sigma_{\mathrm{Cu}}=$ $40.10^{6} \mathrm{~S} / \mathrm{m}, \sigma_{\mathrm{Al}}=28.10^{6} \mathrm{~S} / \mathrm{m}$ and $\sigma_{\mathrm{Ag} . \mathrm{Ink}}=4.10^{6} \mathrm{~S} / \mathrm{m}$. The resistance value decreases rapidly as the thickness of the antenna increases. The copper and aluminium resistance values, $R_{\text {sant }}$, are fairly close and offer interesting performance results at low thickness while the silver ink requires a large thickness, typically, several tens of microns. It is worth mentioning that the thickness and choice of conductor materials have very little impact on the value of serial inductance, $L_{\text {sant }}$.

Figure 7 Variation of inductance, $L_{\text {sant }}$, and resistance, $R_{\text {sant }}$, depending on the thickness, $t$, of the coil antenna for different metallic materials

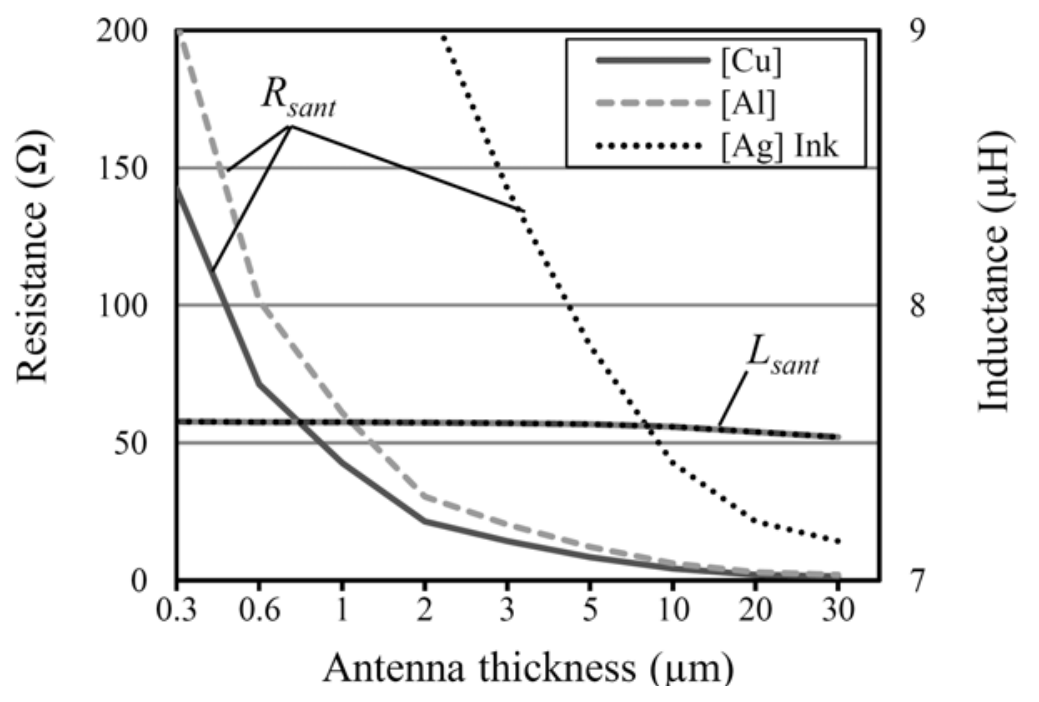




\subsection{Skin depth}

For high frequencies, the electric current flows through the 'skin' of the conductor, at an average depth referred to as 'skin depth'. One defines the skin depth, $\delta$, characterising the penetration of the current in a conductor of thickness $t$ by (Terman, 1943):

$$
\delta=\frac{1}{\sqrt{\pi \mu \sigma f}} \quad(\mathrm{~m})
$$

where $\mu$ is the material permeability, $\sigma$ the conductivity and $f$ the operating frequency. In the HF RFID frequency band: $13.56 \mathrm{MHz}$, the calculated value of the skin depth for aluminium metal is: $\delta=30 \mu \mathrm{m}$ with $\sigma=28.10^{6} \mathrm{~S} / \mathrm{m}$ (experimental value) and $\mu=\mu_{0} \mu_{r}=4 \pi \cdot 10^{-7} \mathrm{H} / \mathrm{m}$ [equation (7)].

There are two distinct cases. Firstly, if the thickness of the conductor is higher than twice the skin depth, the current will flow on the surface of the track and the resistance, $R$, is expressed according to the skin depth. Secondly, as in our case, if the thickness is lower than twice the skin depth $(1 \mu \mathrm{m}<<2 \times 30 \mu \mathrm{m})$, the entire electric current flows through the section of the track and the resistance, $R$, is only expressed as a function of the thickness, $t$, of the conductor as specified in equation (6).

\section{Simulation and measurement results}

The structure of the antenna was simulated using the electromagnetic simulator software, Ansoft Designer. These simulations are used to verify the antenna geometry and to correlate with the previously computed theoretical values. Moreover, the software takes into account the mutual inductance effects of the coils to each other as well as capacitor effects.

The simulator enables, among other things, establishment of antenna impedance, gain, directivity and reflection coefficient between the antenna and the RFID chip values. It is, however, necessary to adjust the design in order to minimise the resistance value and thus satisfy the matching of the impedances real parts.

Graph in Figure 8 represents the reflection coefficient corresponding to the matching. It is given by the respective impedances of the antenna simulated and the RFID chip (Philips Semiconductors, 2006). We notice that the reflection coefficient is minimal in the neighbourhood of $14.4 \mathrm{MHz}$, which means that the chip receives maximum antenna power under optimum operating conditions. However, this reflection coefficient improves as the thickness of the antenna increases, due to the optimisation of matching between the chip and the antenna.

After the theoretical considerations and the tag simulation it is necessary to validate these results experimentally.

\subsection{Thin film characterisations}

\subsubsection{Structural characterisation}

Figure 9 presents the photograph of a prototype HF tag fabricated on paper in the laboratory. External dimensions of inlays are in accordance with standard ISO7810-ID1 
$(85.60 \times 53.98 \mathrm{~mm})$. The antenna has a size of $75 \times 45 \mathrm{~mm}$ with $1 \mu \mathrm{m}$ thick aluminium. The strap with the RFID chip replaces the bridge on the top centre section of the antenna. The thin layer of aluminium is smooth, shiny and silvery. The crystallinity of the aluminium layers is then analysed. Figure 10 compares the X-ray diffraction diagrams of a paper sample without aluminium and the same paper after metallisation of $1 \mu \mathrm{m}$ aluminium thickness. One notes the aluminium peaks with a preferential crystalline orientation along the direction (111) characteristic of the bulk aluminium. This confirms the good structural quality of the Al layer deposited.

Figure 8 Simulation of reflection coefficient between the antenna and the chip depending on the frequency and the antenna thickness, $t$

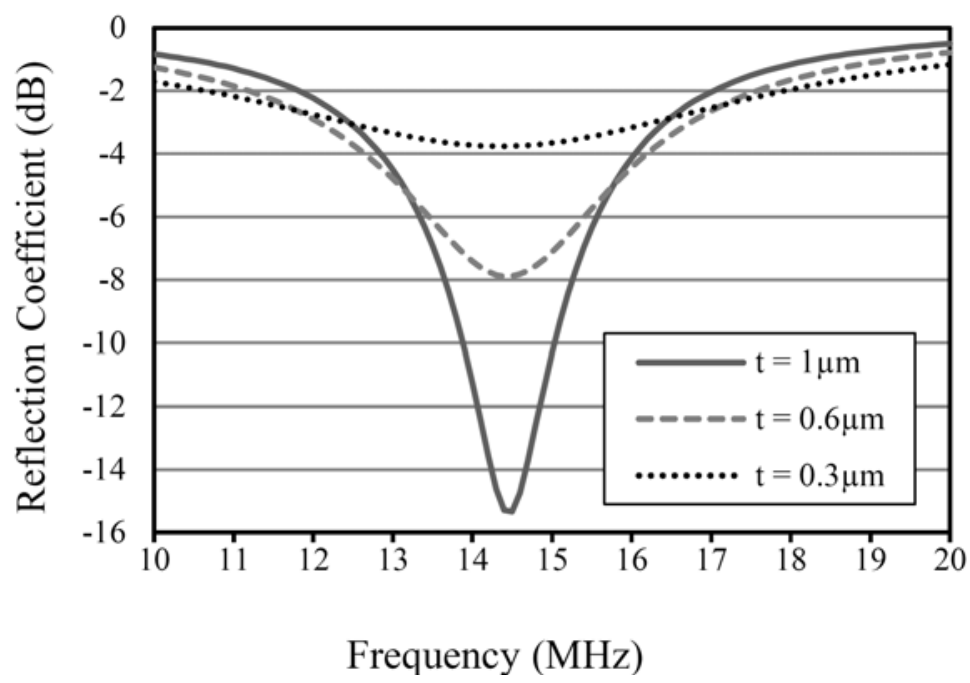

Figure 9 Photograph of a prototype HF tag fabricated on a paper substrate, with overall dimensions of $85.6 \mathrm{~mm} \times 53.98 \mathrm{~mm}$ and $1 \mu \mathrm{m}$ metal thickness (see online version for colours)

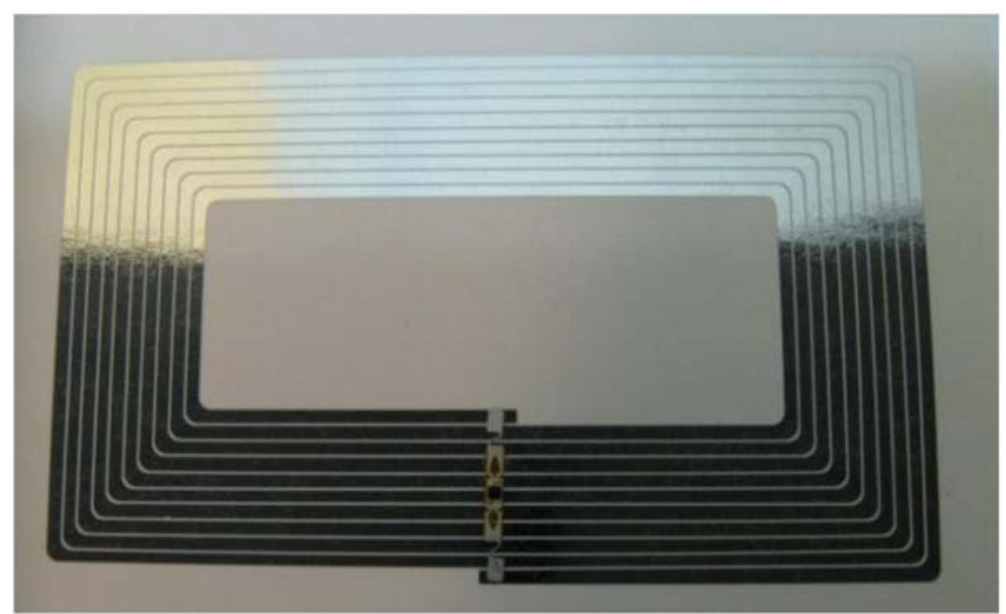


Figure 10 Plot of X-ray diffraction measurement of metalised and un-metalised paper sample based on the diffraction angle

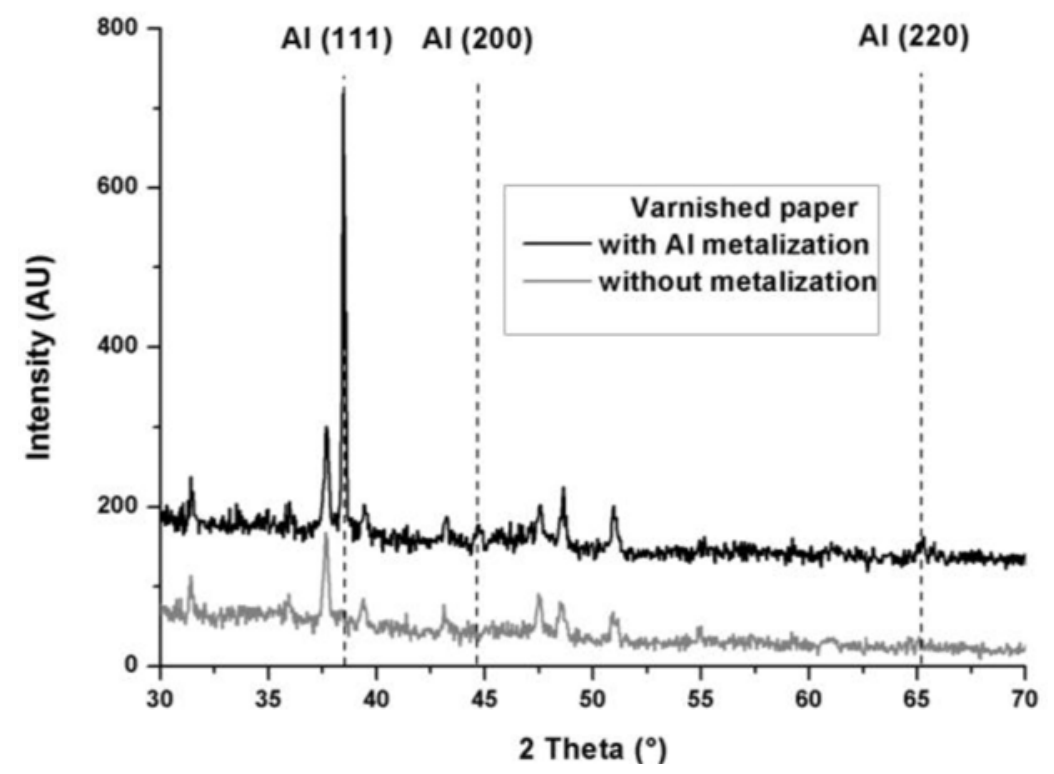

\subsubsection{Physical measurements}

The aluminium thickness, $t$, is measured with a Talystep Veeco Dektak3. Deposit thicknesses range from 300 to $1000 \mathrm{~nm}$ and show variations of 5-10\% between the centre and the edge of the antenna due to the evaporation cone effect, it could weakly impact the antenna impedance.

The resistivity, $\rho$, and the conductivity, $\sigma$, of the film are measured using a four-point probe technique:

$$
\begin{array}{ll}
\rho=R_{s}(\Omega / \text { sq. }) t & (\Omega \mathrm{m}) \\
\sigma=\frac{1}{\rho}=\frac{1}{R s . t} & (\mathrm{~S} . \mathrm{m})
\end{array}
$$

The resistivity, $\rho$, is deducted from equation (8) according to the thickness of the aluminium deposited. For a surface resistance, $R_{\mathrm{s}}$, of $0.12 \Omega / \mathrm{sq}$. and a thickness of $300 \mathrm{~nm}$, the resistivity is equal to $3.6 \times 10^{-8} \Omega . \mathrm{m}$ and therefore the conductivity is $28 \times 10^{6} \mathrm{~S} / \mathrm{m}$ [equation (9)]. This value is close to the known conductivity value of bulk aluminium which is $37.4 \times 10^{6} \mathrm{~S} / \mathrm{m}$. This experimental value is considerably higher than the conductivities obtained with traditional conducting inks used in inkjet printing, the values of which are around $1.6 \times 10^{6} \mathrm{~S} / \mathrm{m}$ (Nikitin et al., 2005). Taking into account the deposited thicknesses $(300-1000 \mathrm{~nm})$, this conductivity value attests to an excellent layer quality. Moreover, as this aluminium layer is thin, it allows obtaining of low costs for antenna production. 


\subsection{Electrical tests}

The electrical measurements of resistance, inductance and quality factor at various frequencies were carried out using a network analyser, HP 8752A and an impedance analyser, HP 4192A. First, the network analyser is used to check the RFID chip or strap impedance with a Zprob SG-500 and a calibration kit developed in the laboratory. We then measure the impedance of the tag antenna alone with analysers. To finish we connect the RFID chip to the tag antenna and measure the resonant frequency of the tag in a band-width ranging from 10 to $20 \mathrm{MHz}$. The results obtained with this device are illustrated in Figures 11 and 12. Each measurement value is compared with the calculated and simulated values of resistance, inductance, quality factor and resonant frequency, respectively, at various thicknesses between 0.2 and $1.2 \mu \mathrm{m}$.

Figure 11 Resistance, $R_{\text {sant }}$, and inductance, $L_{\text {sant }}$, of the tag antenna depending on the antenna thickness

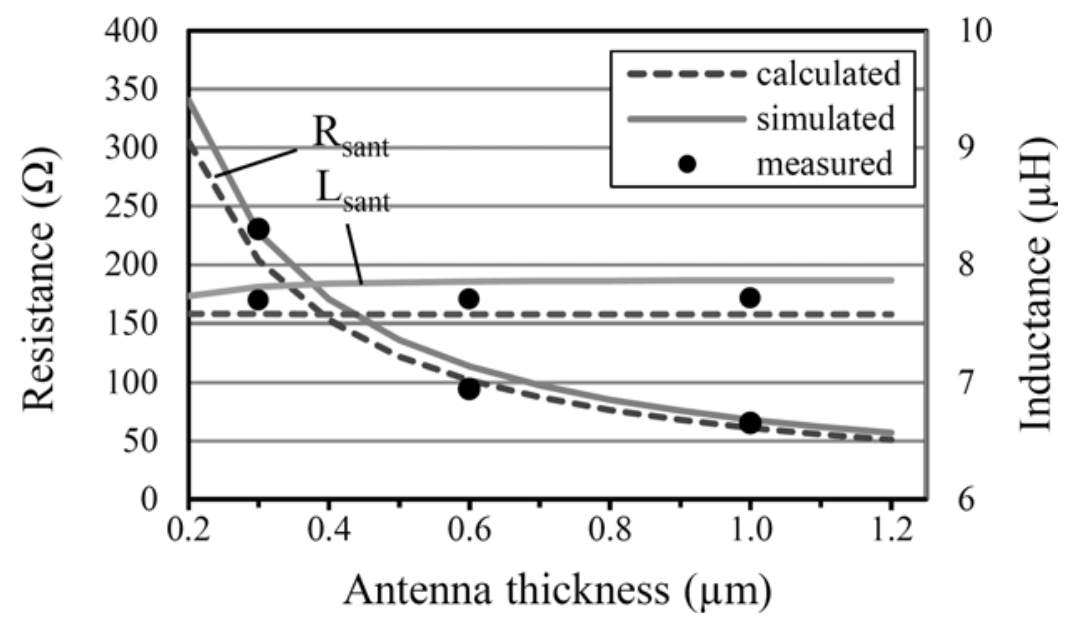

Figure 12 Antenna quality factor and resonant frequency of the tag depending on the antenna thickness

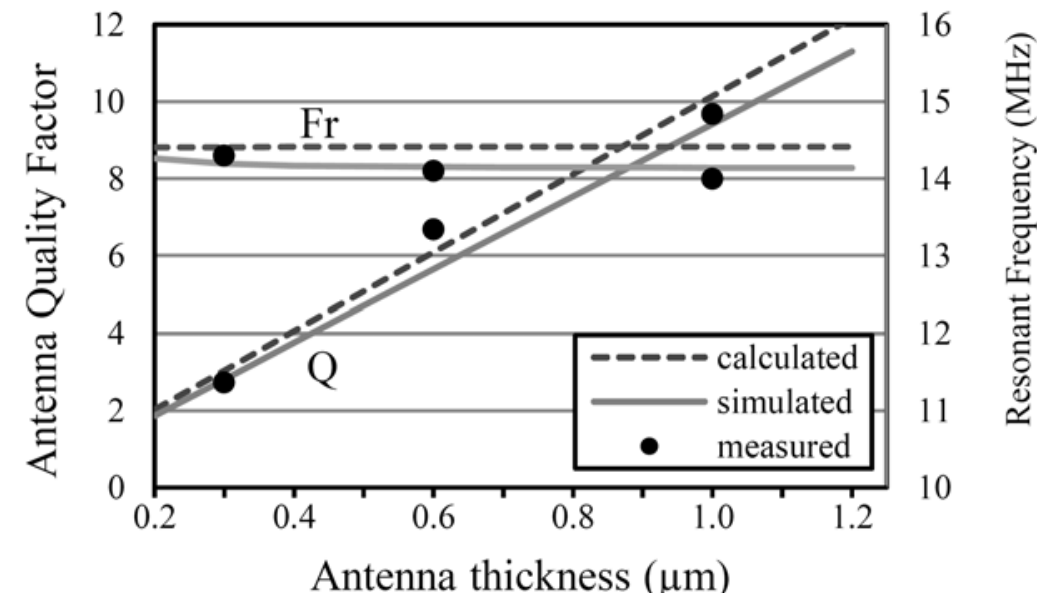


Several prototypes have been produced in aluminium with the following thicknesses: $0.3,0.6$ and $1 \mu \mathrm{m}$. Experimental results are very close to the calculated or simulated values. The differences are mainly due to the difficulties of setting up simulations and the calibration of measuring devices. As for the resistance value, the gap between the calculated and simulated curves includes certainly both radiation resistance and losses. As demonstrated previously, the resistance decreases sharply unlike the inductance which varies slightly depending on the thickness as shown in Figure 11.

The antenna quality factor is proportional to the thickness and the measured values are very close to the calculation and simulation values (Figure 12). Finally, the resonant frequency of the tag function of the equivalent resonant circuit previously measured is only slightly influenced by the thickness of the antenna as shown in Figure 12.

\subsection{HF measurements}

The frequency analysis was carried out using a Tagtester RTS200 from Tagnology Company. This device is composed of a spectrum analyser, an RF generator and an RFID Multi-standard/multi-frequency reader. It allows testing of the operation of RFID tags LF, HF and UHF with two types of RFID measurements: functional tests and electrical tests. After setting the parameters of the device and its antenna, we visualise the resonance frequency, the band-width, the quality factor, the coupling level and finally the transfer rate between the transponder and the Tagtester. Measurements made with the Tagtester were in the same experimental conditions: $2 \mathrm{~cm}$ of distance between measurement antenna and tag, $100 \mathrm{~mW}$ emitted power. These results allow the comparison between laboratory prototypes and two standards HF tags commercially available from UPM Raflatac (Ref 1 and Ref 2). Results are illustrated in Figure 13; the curves represent the coupling level between the Tagtester antennas and the RFID tag under test according to the operating frequency. The higher the coupling level, the better are the matching and the read range of the tag.

Figure 13 Coupling level of tags measured by a Tagtester: laboratory samples $(0.3,0.6$ and $1 \mu \mathrm{m}$ thickness) and two different tags commercially available (Ref 1 and Ref 2)

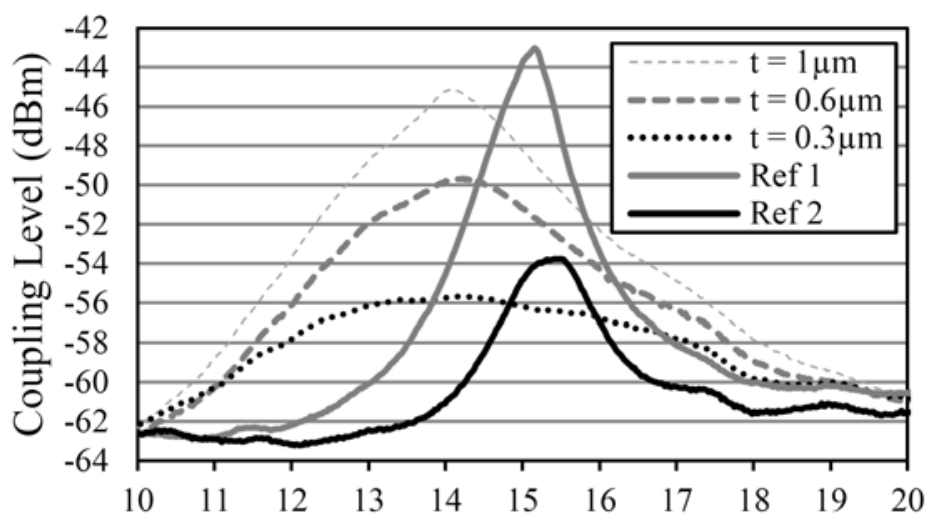

Frequency $(\mathrm{MHz})$ 
Five tags are tested: three prototypes produced in the laboratory with different antenna thickness $(t=1 \mu \mathrm{m} ; 0.6 \mu \mathrm{m} ; 0.3 \mu \mathrm{m})$ and two different tags commercially available, made by $30 \mu \mathrm{m}$ aluminium etching under PET (Ref 1 and Ref 2) as reference. All these tags have the same size and are mounted with the same proximity chip, Mifare ${ }^{\circledR}$, from NXP.

The performance results obtained with the lab prototypes are quite close to the equivalent commercial tags. Concerning the coupling level, the prototypes tags have good levels between $-56 \mathrm{dBm}$ and $-45 \mathrm{dBm} @ 14 \mathrm{MHz}$; commercial tags returns different levels: higher power of $-43 \mathrm{dBm}$ for Ref 1 and lower power of $-54 \mathrm{dBm} @ 15 \mathrm{MHz}$ for Ref 2.

The resonance frequency of prototype tags is a little higher than $13.56 \mathrm{MHz}$ $(14 \mathrm{MHz})$ and their quality factor is low but offers a larger band-width for use in stack configuration. Once again the limited thickness of the antenna does not interfere with tag performance.

After carrying out frequency measurements, we tested the tags performance under true conditions using a standard commercial RFID HF reader (base station) Omnikey ${ }^{\circledR}$ 5553 Desktop reader. It is used for proximity reading and operates with standard chips compatible with ISO14443A/B and 15693. The prototype tags are detected at the maximum read range of the desktop reader $(4 \mathrm{~cm})$. This confirms the feasibility of HF RFID tags made by thermal evaporation for proximity applications.

\section{Conclusion}

For the first time the feasibility of potentially low-cost HF transponders made of paper substrate with a new process in the RFID domain has been proven. In fact, transponders are directly produced on paper substrate with a coil antenna made of a thin layer of aluminium using a thermal evaporation process. Tag performance is measured and checked by calculations and simulations. Despite the low thickness of the antenna metal layer $(\leq 1 \mathrm{um}$ ), the conductivity of aluminium is sufficient to produce functional RFID tags. These have been tested successfully with a commercial proximity reader and a Tagtester RTS200.

Prototypes are functional and easily detected by the reader; the next step is to optimise the design for each family of RFID chips. This will significantly improve performance while maintaining the same low-cost technology on paper.

Effectively, with the help of the use of paper and low amount of metal, this process permits a cost reduction estimated to $20 \%$ compared to equivalent commercial tag.

Moreover, we are currently working with several partners to demonstrate and industrialise our process in mass production using existing industrial solution like metaliser.

We are also currently working on the adaptability of this same process for the production of UHF tags. This work will give rise to another publication. 


\section{References}

Eder, F., Klauk, H., Klauk, M., Zschieschang, U., Schmid, G. and Dehm, C. (2004) 'Organic electronics on paper', Applied Physics Letters, Vol. 84, No. 14, pp.2673-2675.

Elsherbeni, T., ElMahgoub, K., Sydänheimo, L., Ukkonen, L., Elsherbeni, A. and Yang, F. (2010) 'Laboratory scale fabrication techniques for passive UHF RFID tags', Proceedings of the 2010 IEEE Antennas and Propagation Society International Symposium, Toronto, Ontario, Canada, pp.1-4.

Ferrer-Vidal, A., Rida, A., Basat, S., Yang, L. and Tentzeris, M. (2006) 'Integration of sensors and RFID's on ultra-low-cost paper-based substrates for wireless sensor networks applications', Proceedings of the 2006 2nd Workshop on Wireless Sensor Network, Washington, DC, pp.126-128.

Finkenzeller, K. (2003) RFID Handbook, Wiley, Chichester, UK, pp.160-80.

Fortunato, E. and Correia, N. (2008) 'High-performance flexible hybrid field-effect transistors based on cellulose fiber paper', IEEE Electron Device Letters, Vol. 29, No. 9, pp.988-990.

Hunt, D. (2007) RFID - A Guide to Radio Frequency Identification, Wiley, Hoboken, NJ, pp.7-15.

Ludwig, R. (2005) 'Vacuum web coating - state of the art and potential for electronics', Proceedings of the IEEE, Vol. 93, No. 8, pp.1483-1490.

Merilampi, S., Ukkonen, L., Sydänheimo, L., Ruuskanen, P. and Kivikoski, M. (2007) 'Analysis of silver ink bow-tie RFID tag antennas printed on paper substrates', International Journal of Antennas and Propagation, Vol. 2007, p.9.

Nikitin, P., Lam, S. and Rao, K. (2005) 'Low cost silver ink RFID tag antennas', Proceedings of the 2005 IEEE Antennas and Propagation Society International Symposium, Washington, DC, pp.353-356.

Olbrich, P. (2005) 'Advanced coating technology for film capacitor applications', CARTS EUROPE 2004: 18th Annual Capacitor and Resistor Technology Symposium, 18-21 October, Nice, France.

Paret, D. (2003) Applications en identification radiofréquences et cartes à puce sans contact, Dunod, Paris, pp.112-120.

Philips Semiconductors (2006) 'Application note for Mifare ${ }^{\circledR}$, (Card) Coil Design Guide', Product specification, Rev. 3.2.

Sze, S.M. (2001) Semiconductor Devices: Physics and Technology, Hoboken, NJ.

Terman, F.E. (1943) Radio Engineers' Handbook, McGraw-Hill, New York.

Umrath, W. (1998) Fundamentals of Vacuum Technology, Leybold Vacuum, pp.133-135.

Wager, P.A., Eugster, M., Hilty, L.M. and Som, C. (2005) 'Smart labels in municipal solid waste - a case for the precautionary principle?', Environmental Impact Assessment Review, Vol. 25, No. 5, pp.567-586.

Yang, L., Rida, A., Vyas, R. and Tentzeris, M. (2007) 'RFID tag and RF structures on paper substrate using inkjet-printing technology', IEEE Transactions on Microwave Theory and Techniques, Vol. 55, No. 2, pp.2894-2901. 